\title{
Surgical Resection of a Rare Undifferentiated Pancreatic Carcinoma with Osteoclast-Like Giant Cells: A Case Review
}

\author{
Jessica L. Buicko, d, Anne Marie Lopez ${ }^{\mathrm{b}}$, Miguel A. Lopez-Viego ${ }^{\mathrm{c}}$
}

\begin{abstract}
Undifferentiated (anaplastic) pancreatic carcinoma with osteoclastlike giant cells is an extremely rare malignancy with frequency of $0.2 \%$ of reported pancreatic carcinomas. As a whole, undifferentiated pancreatic carcinomas have worse prognoses than poorly differentiated pancreatic adenocarcinomas, yet it is thought that the subtype with osteoclast-like giant cells may in fact have a more promising outlook. We present an unusual case of an undifferentiated pancreatic carcinoma with osteoclast-like giant cells in a 70 -year-old male patient presenting with abdominal pain, nausea, and weight loss, which was treated with surgical resection. This diagnosis is a difficult one as often times presenting symptoms are nonspecific. Endoscopy with biopsy specimens may not be sufficient for a histological diagnosis. The definitive diagnosis and treatment is excision of the mass. Patients often present with advanced disease and complete resection can rarely be preformed. Overall, the prognosis of this subtype of tumor is most similar to common ductal pancreatic carcinomas as opposed to undifferentiated pancreatic carcinomas.
\end{abstract}

Keywords: Pancreas; Pancreatic cancer; Undifferentiated pancreatic cancer; Giant cell tumors

\section{Introduction}

Undifferentiated carcinoma of the pancreas is a rare and extremely aggressive tumor, which has been found to account for $2-7 \%$ of all pancreatic cancers [1]. There are three ma-

\footnotetext{
Manuscript accepted for publication November 15, 2012

${ }^{a}$ University of Miami Miller School of Medicine, Miami, FL, USA

${ }^{\mathrm{b}}$ University of Michigan, BS Cellular and Molecular Biology (2014), Ann Arbor, MI, USA

${ }^{c}$ Department of General and Vascular Surgery, JFK Medical Center, Atlantis, FL and Bethesda Memorial Hospital, Boynton Beach, FL, USA

${ }^{\mathrm{d}}$ Corresponding author: Jessica L. Buicko, University of Miami Miller School of Medicine, Miami, FL, USA.

Email: JBuicko@med.miami.edu
}

doi: http://dx.doi.org/10.4021/jmc1005e jor subtypes of undifferentiated carcinoma of the pancreas that include: giant cell carcinoma, spindle cell carcinoma, and round cell carcinoma $[1,2]$. The giant cell carcinoma subtype can be further divided on a histological basis into two distinct categories that include: pleomorphic giant cell, and osteoclast-like giant cell tumors. The first osteoclast-like giant cell tumor was described by Rosai et al in 1968 [2]. As of 2006 , there have been 60 cases of these tumors reported in the literature [3]. This subtype is estimated to comprise $0.2 \%$ of the total number of pancreatic carcinomas [4]. We present an unusual case of an undifferentiated carcinoma with osteoclast-like giant cells of the pancreas in a 70-year-old male patient presenting with abdominal pain, nausea, and weight loss, which was treated with surgical resection.

\section{Case Report}

A 70-year-old Hispanic male presented to our teaching service with a three-month history of abdominal pain associated with nausea, vomiting, and an unintentional weight loss of approximately 30 pounds. His medical history was significant for diabetes mellitus and gastroesophageal reflux disease. He was originally admitted for acute pancreatitis. A CT scan of the abdomen and pelvis was performed that showed an abnormal heterogeneous enlargement of the pancreatic head measuring $4.9 \times 3.2 \mathrm{~cm}$ (Fig. 1). In addition, there was a $2.9 \mathrm{~cm}$ cyst along the pancreatic head and descending portion of the duodenum. There was atrophy of the remainder of the pancreas. The patient subsequently underwent endoscopic ultrasound and fine-needle biopsy, which showed a poorly differentiated carcinoma. An underlying lymphoma could not be excluded and because of this, a second biopsy was performed that revealed the same findings as the original biopsy. The patient was then referred to our service and the surgical team proceeded with a pancreaticoduodenectomy without complications.

On gross examination, the removed portion of the pancreas measured $5.5 \times 3.2 \mathrm{~cm}$. Microscopic examination revealed a malignant tumor with a diameter of $2.1 \mathrm{~cm}$ with a heterogeneous histologic appearance. A large portion of the tumor was composed of spindle cells with a fasciculated 


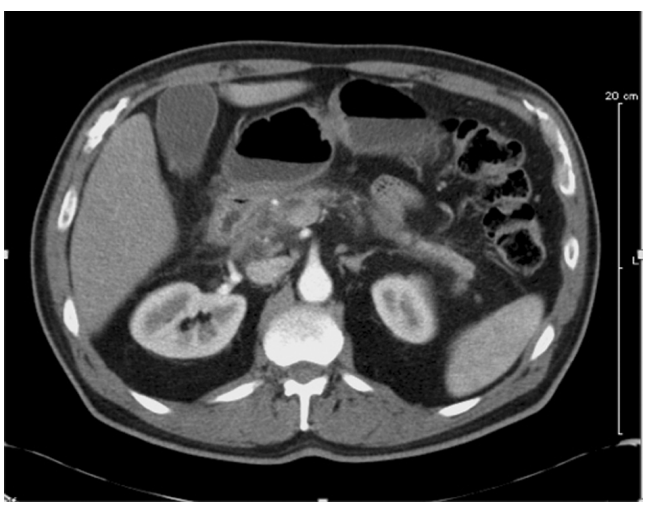

Figure 1. CT Scan showing prominent mass at head of the pancreas with associated biliary and pancreatic duct dilatation. Atrophy of the body and tail is seen, along with peripancreatic lymph node involvement.

architecture. The tumor cells resembled neoplastic smooth muscle cells with a mitotic index of approximately 6 mitosis per 10 high power fields. In other sections of the tumor, there was substantial necrosis and a different histologic appearance as there were many multinucleated osteoclast-like giant cells (Fig. 2). This portion did not have a fasciculated architecture as in the other section.

Immunohistochemistry revealed that the mononuclear cells were positive for SMA, CD 99, Calponin, and EMA. There were a few cells that expressed S-100. The osteoclastlike giant cells are positive for CD-68 and KP-1. CD 117, Pankeratin, Desmin, Ber-EP4, MOC-31, and TAG 72.3 were negative. Eleven benign lymph nodes were present.

Based on morphology and immunohistochemistry, this tumor was classified by the pathology department as an undifferentiated carcinoma with osteoclast-like giant cells (Fig. $3,4)$.

\section{Discussion}

This case of an undifferentiated pancreatic carcinoma with

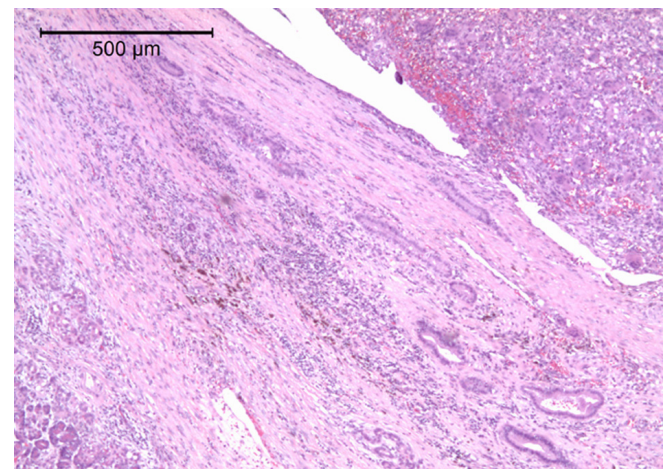

Figure 2. Low-Powered View Demonstrating Region of Osteoclast-like Giant Cells.

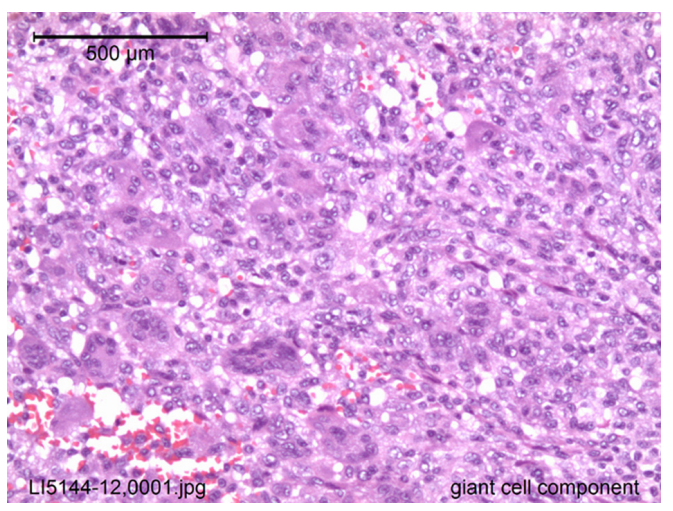

Figure 3. High-Powered View of Giant Cell Component.

osteoclast-like giant cells is extremely rare, with limited information found in the current literature, most of which consists of case reports. These tumors have a controversial histogenesis and there have been debates in the current literature as to their true origin. Several authors believe that the tumors have an epithelial origin whereas others believe the tumors to have a mesenchymal derivation [5]. Our cases support this notion as the tumor was found to have a very heterogenous structure with both sarcomatoid and giant cell components.

From a clinical standpoint, the most common presenting symptoms are abdominal pain and weight loss. Jaundice can also be seen if the tumor is located at the head of the pancreas [3]. In their literature review of fifty-four cases reported, Osaka et al found that there were 25 men and 29 women with a median age of 61.9 diagnosed with this type of tumor. The median size was $10.1 \mathrm{~cm}$ [4]. Most of the osteoclasticlike giant cell tumors are found in the head of the pancreas. They exhibit rapid tumor growth. Bauditz et al report that in the fifty documented cases in which the tumor size was measured, only three cancers measured under $3 \mathrm{~cm}$. In the remaining 47 cases, $80 \%$ were greater than $5 \mathrm{~cm}$, and $50 \%$ greater than $10 \mathrm{~cm}$ [3]. Our patient's tumor measured $2.1 \mathrm{~cm}$ in diameter.

The differential diagnosis of undifferentiated pancreatic

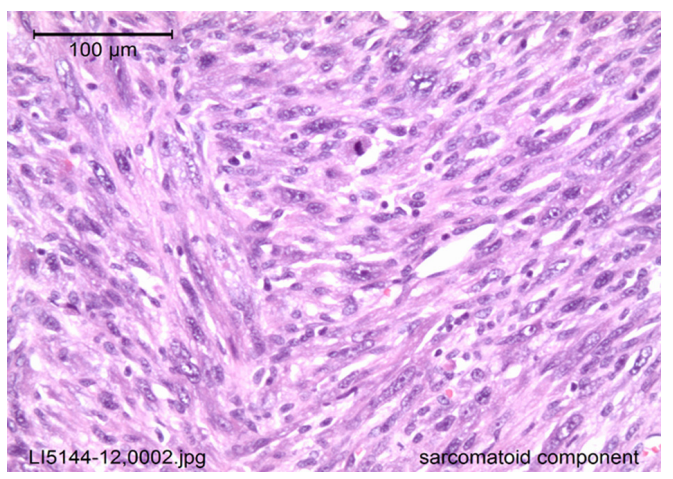

Figure 4. High-Powered View of Sarcomatoid Component. 
carcinomas with osteoclastic-like giant cells can include: pancreatic cystadenomas, cystadenocarcinomas, serous and mucinous cystic tumors, pancreatic pseudocysts, ductal pancreatic carcinomas, and neuroendocrine tumors [3]. Immunohistochemically, CD68 and cytokeratin are recommended to subtype the tumor. Giant cells are strongly immunoreactive to CD68 and confirm their osteoclastic nature. Cytokeratin shows positivity in normal pancreatic tissue and therefore these cells are negative for cytokeratin [6]. Desmin, calretin, calponin, Bcl2, CD34, pancytokeratin, and E-cadherin also stain negatively within the tumor cells [7].

The prognosis of undifferentiated carcinoma of the pancreas is usually worse than that of poorly differentiated ductal pancreatic carcinoma. Poorly differentiated ductal adenocarcinoma is known for having a dismal prognosis and in general, the prognosis for undifferentiated pancreatic cancer is even worse [1]. Nonetheless, there is some evidence to support that the specific subtype of undifferentiated carcinoma of the pancreas with osteoclast-like giant cells may be associated with increased long-term survival. Maksymov et al noted that their patient remained clinically and radiologically disease free after 36 months [1].

In regards to treatment, surgery remains the first-line in most cases if the tumor is resectable [6]. The metastatic spread of undifferentiated carcinoma of the pancreas with osteoclast-like giant cells is slow. Osaka et al reported that of the twenty patients in their review with unresectable cancer, all were dead within ten months. In stark contrast, if the tumor was thought to be resectable, $70 \%$ of the patients were alive more than 1 year after the treatment [4]. This data suggests that the resectability of the tumor is most closely associated with prognosis.

\section{Conclusion}

Undifferentiated carcinoma of the pancreas with osteoclastlike giant cells is extremely rare malignancy with a limited number of reported cases in the literature. Diagnosis is best achieved by combining radiological studies with immunohistochemistry of the excised tumor. More long-term data is needed to follow patients with undifferentiated carcinoma of the pancreas with osteoclast-like giant cells to adequately predict long-term survival. This may prove to be a difficult task considering the rarity of this tumor.

\section{Special Thanks}

Dr. Gary V. Kuehl, MD. Bethesda Memorial Hospital Pathology Dept.

\section{References}

1. Maksymov V, Khalifa MA, Bussey A, Carter B, Hogan M. Undifferentiated (anaplastic) carcinoma of the pancreas with osteoclast-like giant cells showing various degree of pancreas duct involvement. A case report and literature review. JOP. 2011;12(2):170-176.

2. Schaffner TJ, Richmond BK. Osteoclast-like giant cell tumor of the pancreas with subsequent pulmonary metastases. Am Surg. 2011;77(12):E275-277.

3. Bauditz J, Rudolph B, Wermke W. Osteoclast-like giant cell tumors of the pancreas and liver. World J Gastroenterol. 2006;12(48):7878-7883.

4. Osaka H, Yashiro M, Nishino H, Nakata B, Ohira M, Hirakawa K. A case of osteoclast-type giant cell tumor of the pancreas with high-frequency microsatellite instability. Pancreas. 2004;29(3):239-241.

5. Loya AC, Ratnakar KS, Shastry RA. Combined osteoclastic giant cell and pleomorphic giant cell tumor of the pancreas: a rarity. An immunohistochemical analysis and review of the literature. JOP. 2004;5(4):220-224.

6. Mannan R, Khanna M, Bhasin TS, Misra V, Singh PA. Undifferentiated carcinoma with osteoclast-like giant cell tumor of the pancreas: a discussion of rare entity in comparison with pleomorphic giant cell tumor of the pancreas. Indian J Pathol Microbiol. 2010;53(4):867-868.

7. Rauramaa T, Pulkkinen J, Miettinen P, Kainulainen S, Seppa A, Karja V. Case report: osteoclast-like giant cell tumour of the pancreas without epithelial differentiation. J Clin Pathol. 2010;63(4):376-377. 